Pacific

Journal of

Mathematics

MÖBIUS ISOPARAMETRIC HYPERSURFACES WITH THREE DISTINCT PRINCIPAL CURVATURES

ZeJun Hu AND Deying Li

Volume 232 No. 2

October 2007 


\title{
MÖBIUS ISOPARAMETRIC HYPERSURFACES WITH THREE DISTINCT PRINCIPAL CURVATURES
}

\author{
ZEJUN HU AND DEYING LI \\ Dedicated to Professor Anmin Li on his sixtieth birthday
}

\begin{abstract}
We investigate Möbius isoparametric hypersurfaces in the $(n+1)$-Euclidean unit sphere $\mathbb{S}^{n+1}$ with three distinct Möbius principal curvatures. As direct consequence of our main result, we establish the complete classification for all such hypersurfaces in $\mathbb{S}^{6}$.
\end{abstract}

\section{Introduction}

Let $x: M^{n} \rightarrow \mathbb{S}^{n+1}$ be a hypersurface in the $(n+1)$-dimensional unit sphere $\mathbb{S}^{n+1}$ without umbilic point. We choose a local orthonormal basis $\left\{e_{1}, \cdots, e_{n}\right\}$ with respect to the induced metric $I=d x \cdot d x$ and the dual basis $\left\{\theta_{1}, \cdots, \theta_{n}\right\}$. Let $h=\sum_{i, j} h_{i j} \theta_{i} \otimes \theta_{j}$ be the second fundamental form of $x$, with squared length $\|h\|^{2}=\sum_{i, j}\left(h_{i j}\right)^{2}$ and mean curvature $H=\frac{1}{n} \sum_{i} h_{i i}$, respectively. Define $\rho^{2}=$ $n /(n-1) \cdot\left(\|h\|^{2}-n H^{2}\right)$. Then the positive definite form $g=\rho^{2} d x \cdot d x$ is a Möbius invariant and is called the Möbius metric of $x: M^{n} \rightarrow \mathbb{S}^{n+1}$. The Möbius second fundamental form $\mathbf{B}$, another basic Möbius invariant of $x$, together with $g$ determine completely a hypersurface of $\mathbb{S}^{n+1}$ up to Möbius equivalence; see Theorem 2.2.

An important class of hypersurfaces for Möbius differential geometry consists of the so-called Möbius isoparametric hypersurfaces in $\mathbb{S}^{n+1}$. Recall that, according to [Li et al. 2002], a Möbius isoparametric hypersurface of $\mathbb{S}^{n+1}$ is an umbilic-free hypersurface of $\mathbb{S}^{n+1}$ such that the Möbius invariant 1-form

$$
\Phi=-\rho^{-1} \sum_{i}\left\{e_{i}(H)+\sum_{j}\left(h_{i j}-H \delta_{i j}\right) e_{j}(\log \rho)\right\} \theta_{i}
$$

vanishes and all of its Möbius principal curvatures are constant. Note that by Möbius principal curvatures, we mean the so-called eigenvalues of the Möbius

MSC2000: primary 53A30; secondary 53B25.

Keywords: Möbius isoparametric hypersurface, Möbius equivalence, Möbius second fundamental form, Möbius metric, Möbius form.

Project supported by grants of NSFC (No. 10671181), DAAD and SRF for ROCS, SEM.. 
shape operator $\Psi:=\rho^{-1}(\mathbf{S}-H$ id) with respect to $g$; here $\mathbf{S}$ denotes the shape operator of $x: M^{n} \rightarrow \mathbb{S}^{n+1}$. This definition of Möbius isoparametric hypersurfaces is meaningful when compared with that of (Euclidean) isoparametric hypersurfaces in $\mathbb{S}^{n+1}$. The images of all hypersurfaces of the sphere with constant mean curvature and constant scalar curvature under Möbius transformation satisfy $\Phi \equiv 0$, and the Möbius invariant operator $\Psi$ plays the same role in Möbius geometry as $\mathbf{S}$ does in Euclidean geometry (see Theorem 2.2). Standard examples of Möbius isoparametric hypersurfaces are the images of (Euclidean) isoparametric hypersurfaces in $\mathbb{S}^{n+1}$ under Möbius transformations, but there are other examples that cannot be obtained in this way. For example, they occur in our classification for hypersurfaces of $\mathbb{S}^{n+1}$ with parallel Möbius second fundamental form; this means that the Möbius second fundamental form is parallel with respect to the Levi-Civita connection of the Möbius metric $g$. See [Li et al. 2002; Hu and Li 2004] for details. On the other hand, it was proved in [Li et al. 2002] that any Möbius isoparametric hypersurface is in particular a Dupin hypersurface, which implies from [Thorbergsson 1983] that for a compact Möbius isoparametric hypersurface embedded in $\mathbb{S}^{n+1}$, the number $\gamma$ of distinct principal curvatures can only take the values $\gamma=2,3,4,6$.

Li et al. [2002] classified locally all Möbius isoparametric hypersurfaces of $\mathbb{S}^{n+1}$ with $\gamma=2$. By relaxing the restriction of $\gamma=2$, $\mathrm{Hu}$ and $\mathrm{Li}$ [2005a] and $\mathrm{Hu}$ et al. [2007] classified all Möbius isoparametric hypersurfaces in $\mathbb{S}^{4}$ and $\mathbb{S}^{5}$, respectively. To be precise, they showed that a Möbius isoparametric hypersurface in $\mathbb{S}^{4}$ is either of parallel Möbius second fundamental form or Möbius equivalent to the Euclidean isoparametric hypersurface in $\mathbb{S}^{4}$ with three distinct principal curvatures, that is, a tube of constant radius over a standard Veronese embedding of $\mathrm{RP}^{2}$ into $\mathbb{S}^{4}$. However, a hypersurface in $\mathbb{S}^{5}$ is Möbius isoparametric if and only if it satisfies either of two properties: First, it has parallel Möbius second fundamental form or is Möbius equivalent to the preimage of the stereographic projection of the cone $\tilde{x}: N^{3} \times \mathbb{R}^{+} \rightarrow \mathbb{R}^{5}$ defined by $\tilde{x}(x, t)=t x$, where $t \in \mathbb{R}^{+}$and $x: N^{3} \rightarrow \mathbb{S}^{4} \hookrightarrow \mathbb{R}^{5}$ is the Cartan isoparametric immersion in $\mathbb{S}^{4}$ with three principal curvatures. Second, it is Möbius equivalent to the Euclidean isoparametric hypersurfaces in $\mathbb{S}^{5}$ with four distinct principal curvatures. All these results remind us of their counterparts in Dupin hypersurfaces; see [Thorbergsson 1983; Pinkall 1985; Niebergall 1991; 1992; Cecil and Jensen 1998; 2000].

Because all Möbius isoparametric hypersurfaces have been classified both for $n \leq 4$ in [Hu and Li 2005a; Hu et al. 2007] and for all $n \geq 2$ with two distinct Möbius principal curvatures in [Li et al. 2002], we will consider here Möbius isoparametric hypersurfaces $M^{n}$ in $\mathbb{S}^{n+1}$ with three distinct Möbius principal curvatures for all $n \geq 5$. For further background, we note that the classification of all such hypersurfaces under the Möbius transformation group equivalence can 
be compared with that of the Dupin hypersurfaces with three principal curvatures under the Lie sphere transformation group equivalence established by Cecil and Jensen [1998]. We find it interesting that the Lie sphere transformation group contains the Möbius transformation group in $\mathbb{S}^{n+1}$ as a subgroup and the dimension difference is $n+3$. Therefore, the Möbius differential geometry for hypersurfaces in spheres seems essentially different from the Lie sphere geometry and therefore merits more attention.

Nevertheless, for simplicity, we focus on when one of the three distinct Möbius principal curvatures has multiplicity one. For Möbius isoparametric hypersurfaces with nonparallel Möbius second fundamental form and three distinct Möbius principal curvatures - all of which have multiplicity not smaller than two - the classification is much more involved and will be given in a forthcoming paper.

We will establish these classification results:

Main Theorem. Let $x: M^{n} \rightarrow \mathbb{S}^{n+1}(n \geq 5)$ be a Möbius isoparametric hypersurface with three distinct Möbius principal curvatures such that one of them is simple. Then $x$ is Möbius equivalent to an open part of one of these hypersurfaces in $\mathbb{S}^{n+1}$ :

(i) The preimage of the stereographic projection of the warped product embedding

$$
\tilde{x}: \mathbb{S}^{p}(a) \times \mathbb{S}^{q}\left(\sqrt{1-a^{2}}\right) \times \mathbb{R}^{+} \times \mathbb{R}^{n-p-q-1} \rightarrow \mathbb{R}^{n+1},
$$

with $p \geq 1, q \geq 1, p+q \leq n-1$, and $0<a<1$, defined by

$$
\tilde{x}\left(u^{\prime}, u^{\prime \prime}, t, u^{\prime \prime \prime}\right)=\left(t u^{\prime}, t u^{\prime \prime}, t u^{\prime \prime \prime}\right) \text {, }
$$

where $u^{\prime} \in \mathbb{S}^{p}(a), u^{\prime \prime} \in \mathbb{S}^{q}\left(\sqrt{1-a^{2}}\right), t \in \mathbb{R}^{+}$, and $u^{\prime \prime \prime} \in \mathbb{R}^{n-p-q-1}$.

(ii) Minimal hypersurfaces defined by

$$
\tilde{x}=\left(\tilde{x}_{1}, \tilde{x}_{2}\right): \tilde{M}^{n}=N^{3} \times \mathbb{H}^{n-3}\left(-\frac{n-1}{6 n}\right) \rightarrow \mathbb{S}^{n+1},
$$

where $\tilde{x}_{1}=y_{1} / y_{0}, \tilde{x}_{2}=y_{2} / y_{0}$, with $y_{0} \in \mathbb{R}^{+}, y_{1} \in \mathbb{R}^{5}, y_{2} \in \mathbb{R}^{n-3}$. Here $y_{1}$ : $N^{3} \rightarrow \mathbb{S}^{4}(\sqrt{6 n /(n-1)}) \hookrightarrow \mathbb{R}^{5}$ is Cartan's minimal isoparametric hypersurface with vanishing scalar curvature and principal curvatures $\pm \sqrt{(n-1) / 2 n}$, 0. Also $\left(y_{0}, y_{2}\right): \mathbb{1}^{n-3}(-(n-1) / 6 n) \hookrightarrow \mathbb{L}^{n-2}$ is the standard embedding of the hyperbolic space of sectional curvature $-(n-1) / 6 n$ into the $(n-2)$ dimensional Lorentz space with $-y_{0}^{2}+y_{2}^{2}=-6 n /(n-1)$.

If $n=5$, then hypersurfaces with three distinct Möbius principal curvatures trivially satisfy the assumption that at least one of the principal curvatures is simple. Hence we have immediately: 
Corollary 1.1. Let $x: M^{5} \rightarrow \mathbb{S}^{6}$ be a Möbius isoparametric hypersurface with three distinct Möbius principal curvatures. Then $x$ is Möbius equivalent to an open part of one of these hypersurfaces in $\mathbb{S}^{6}$ :

(i) The preimage of the stereographic projection of the warped product embedding

$$
\tilde{x}: \mathbb{S}^{p}(a) \times \mathbb{S}^{q}\left(\sqrt{1-a^{2}}\right) \times \mathbb{R}^{+} \times \mathbb{R}^{4-p-q} \rightarrow \mathbb{R}^{6},
$$

with $p \geq 1, q \geq 1, p+q \leq 4$, and $0<a<1$, defined by

$$
\tilde{x}\left(u^{\prime}, u^{\prime \prime}, t, u^{\prime \prime \prime}\right)=\left(t u^{\prime}, t u^{\prime \prime}, t u^{\prime \prime \prime}\right),
$$

where $u^{\prime} \in \mathbb{S}^{p}(a), u^{\prime \prime} \in \mathbb{S}^{q}\left(\sqrt{1-a^{2}}\right), t \in \mathbb{R}^{+}, u^{\prime \prime \prime} \in \mathbb{R}^{4-p-q}$.

(ii) Minimal hypersurfaces defined by

$$
\tilde{x}=\left(\tilde{x}_{1}, \tilde{x}_{2}\right): \tilde{M}^{5}=N^{3} \times \mathbb{H}^{2}\left(-\frac{2}{15}\right) \rightarrow \mathbb{S}^{6}
$$

with $\tilde{x}_{1}=y_{1} / y_{0}, \tilde{x}_{2}=y_{2} / y_{0}$, where $y_{0} \in \mathbb{R}^{+}, y_{1} \in \mathbb{R}^{5}, y_{2} \in \mathbb{R}^{2}$. Here $y_{1}: N^{3} \rightarrow \mathbb{S}^{4}\left(\sqrt{30} / 2 \hookrightarrow \mathbb{R}^{5}\right.$ is Cartan's minimal isoparametric hypersurface with vanishing scalar curvature and principal curvatures $\pm \sqrt{10} / 5,0$, and $\left(y_{0}, y_{2}\right): \mathbb{\boxplus}^{2}(-2 / 15) \hookrightarrow \mathbb{L}^{3}$ is the standard embedding of the hyperbolic space of sectional curvature -2/15 into the 3-dimensional Lorentz space with $-y_{0}^{2}+y_{2}^{2}=-15 / 2$.

Remark 1.2. The hypersurfaces in (i) consist of two families both of which are of parallel Möbius second fundamental form, whereas (ii) has only one hypersurface whose Möbius second fundamental form is not parallel.

This paper has four more sections. In Section 2, we first review some elementary facts of Möbius geometry for hypersurfaces in $\mathbb{S}^{n+1}$, and then we present classifications for hypersurfaces of $\mathbb{S}^{n+1}$ with parallel Möbius second fundamental form and for those with two distinct constant Blaschke eigenvalues. These classifications have been achieved in [Hu and Li 2004] and [Li and Zhang 2007], respectively. In Section 3, by investigating Möbius isoparametric hypersurfaces of $\mathbb{S}^{n+1}$ with nonparallel Möbius second fundamental form and having three distinct Möbius principal curvatures, one of which is simple, we show its Möbius principal curvature must be zero with multiplicity $n-2$. Furthermore, we prove Theorem 3.7, which gives a preliminary classification for such hypersurfaces. In Section 4, we prove Proposition 4.2, Proposition 4.5 and Proposition 4.6 by calculating the Möbius invariants of the hypersurfaces appearing in Theorem 3.7. Finally, in Section 5, we complete the proof of Main Theorem. 


\section{Möbius invariants for hypersurfaces in $\mathbb{S}^{n+1}$}

Here, we define Möbius invariants and recall structure equations for hypersurfaces in $\mathbb{S}^{n+1}$. For more detail, see [Wang 1998]. Let $\mathbb{L}^{n+3}$ be the Lorentz space, namely $\mathbb{R}^{n+3}$ with inner product $\langle\cdot, \cdot\rangle$ defined by

$$
\langle x, w\rangle=-x_{0} w_{0}+x_{1} w_{1}+\cdots+x_{n+2} w_{n+2}
$$

for $x=\left(x_{0}, x_{1}, \cdots, x_{n+2}\right)$ and $w=\left(w_{0}, w_{1}, \cdots, w_{n+2}\right) \in \mathbb{R}^{n+3}$.

Let $x: M^{n} \rightarrow \mathbb{S}^{n+1} \hookrightarrow \mathbb{R}^{n+2}$ be an immersed umbilic-free hypersurface of $\mathbb{S}^{n+1}$. We define the Möbius position vector field $Y: M^{n} \rightarrow \mathbb{L}^{n+3}$ of $x$ by $Y=\rho(1, x)$, where

$$
\rho^{2}=\frac{n}{n-1}\left(\|h\|^{2}-n H^{2}\right)>0 .
$$

Then we have this classical result:

Theorem 2.1 [Wang 1998]. Two hypersurfaces $x, \tilde{x}: M^{n} \rightarrow \mathbb{S}^{n+1}$ are Möbius equivalent if and only if there exists $T$ in the Lorentz group $\mathrm{O}(n+2,1)$ in $\mathbb{L}^{n+3}$ such that $Y=\tilde{Y} T$.

It follows immediately that $g=\langle d Y, d Y\rangle=\rho^{2} d x \cdot d x$ is a Möbius invariant, and it is defined as the Möbius metric of $x: M^{n} \rightarrow \mathbb{S}^{n+1}$.

Let $\Delta$ be the Beltrami-Laplace operator of $g$. Defining

$$
N=-\frac{1}{n} \Delta Y-\frac{1}{2 n^{2}}\langle\Delta Y, \Delta Y\rangle Y,
$$

one can show that

$$
\begin{aligned}
\langle\Delta Y, Y\rangle & =-n, & \langle\Delta Y, d Y\rangle & =0, & \langle\Delta Y, \Delta Y\rangle & =1+n^{2} R, \\
\langle Y, Y\rangle & =0, & \langle N, Y\rangle & =1, & \langle N, N\rangle & =0,
\end{aligned}
$$

where $R$ is the normalized scalar curvature of $g$ and is called the normalized Möbius scalar curvature of $x: M^{n} \rightarrow \mathbb{S}^{n+1}$.

Let $\left\{E_{1}, \cdots, E_{n}\right\}$ be a local orthonormal basis for $\left(M^{n}, g\right)$ with dual basis $\left\{\omega_{1}, \cdots, \omega_{n}\right\}$, and write $Y_{i}=E_{i}(Y)$. Then it follows from (2-1) and (2-2) that

$$
\left\langle Y_{i}, Y\right\rangle=\left\langle Y_{i}, N\right\rangle=0, \quad\left\langle Y_{i}, Y_{j}\right\rangle=\delta_{i j}, \quad 1 \leq i, j \leq n .
$$

Let $V$ be the orthogonal complement to the subspace $\operatorname{span}\left\{Y, N, Y_{1}, \cdots, Y_{n}\right\}$ in $\mathbb{L}^{n+3}$. Then along $M$ we have the orthogonal decomposition

$$
\mathbb{L}^{n+3}=\operatorname{span}\{Y, N\} \oplus \operatorname{span}\left\{Y_{1}, \cdots, Y_{n}\right\} \oplus V,
$$

where $V$ is called the Möbius normal bundle of $x: M^{n} \rightarrow \mathbb{S}^{n+1}$. A local unit vector basis $E=E_{n+1}$ for $V$ can be written as

$$
E=E_{n+1}:=\left(H, H x+e_{n+1}\right) .
$$


Then, along $M^{n},\left\{Y, N, Y_{1}, \cdots, Y_{n}, E\right\}$ forms a moving frame in $\mathbb{L}^{n+3}$. Unless otherwise stated, we will henceforth use the range $1 \leq i, j, k, l, t \leq n$ for indices.

We can write the structure equations as:

$$
\begin{aligned}
d Y & =\sum_{i} Y_{i} \omega_{i}, \\
d N & =\sum_{i, j} A_{i j} \omega_{j} Y_{i}+\sum_{i} C_{i} \omega_{i} E, \\
d Y_{i} & =-\sum_{j} A_{i j} \omega_{j} Y-\omega_{i} N+\sum_{j} \omega_{i j} Y_{j}+\sum_{i} B_{i j} \omega_{j} E, \\
d E & =-\sum_{i} C_{i} \omega_{i} Y-\sum_{i, j} B_{i j} \omega_{j} Y_{i},
\end{aligned}
$$

where $\omega_{i j}$ is the connection form of the Möbius metric $g$ and is defined by the structure equations $d \omega_{i}=\sum_{j} \omega_{i j} \wedge \omega_{j}, \omega_{i j}+\omega_{j i}=0$. The tensors $\mathbf{A}=\sum_{i, j} A_{i j} \omega_{i} \otimes$ $\omega_{j}, \Phi=\sum_{i} C_{i} \omega_{i}$, and $\mathbf{B}=\sum_{i, j} B_{i j} \omega_{i} \otimes \omega_{j}$ are respectively called the Blaschke tensor, the Möbius form, and the Möbius second fundamental form of $x: M^{n} \rightarrow$ $\mathbb{S}^{n+1}$. The relations between $\Phi, \mathbf{B}, \mathbf{A}$ and the Euclidean invariants of $x$ are given by [Wang 1998]:

$$
\begin{aligned}
& C_{i}=-\rho^{-2}\left(e_{i}(H)+\sum_{j}\left(h_{i j}-H \delta_{i j}\right) e_{j}(\log \rho)\right), \\
& B_{i j}=\rho^{-1}\left(h_{i j}-H \delta_{i j}\right), \\
& A_{i j}=-\rho^{-2}\left(\operatorname{Hess}_{i j}(\log \rho)-e_{i}(\log \rho) e_{j}(\log \rho)-H h_{i j}\right) \\
& \quad-\frac{1}{2} \rho^{-2}\left(|\nabla \log \rho|^{2}-1+H^{2}\right) \delta_{i j},
\end{aligned}
$$

where $\operatorname{Hess}_{i j}$ and $\nabla$ are the Hessian matrix and the gradient operator with respect to the orthonormal basis $\left\{e_{i}\right\}$ of $d x \cdot d x$.

The covariant derivatives of $C_{i}, A_{i j}, B_{i j}$ are defined by

$$
\begin{aligned}
\sum_{j} C_{i, j} \omega_{j} & =d C_{i}+\sum_{j} C_{j} \omega_{j i}, \\
\sum_{k} A_{i j, k} \omega_{k} & =d A_{i j}+\sum_{k} A_{i k} \omega_{k j}+\sum_{k} A_{k j} \omega_{k i}, \\
\sum_{k} B_{i j, k} \omega_{k} & =d B_{i j}+\sum_{k} B_{i k} \omega_{k j}+\sum_{k} B_{k j} \omega_{k i} .
\end{aligned}
$$

The integrability conditions for the structure equations (2-3) are

$$
\begin{aligned}
A_{i j, k}-A_{i k, j} & =B_{i k} C_{j}-B_{i j} C_{k}, \\
C_{i, j}-C_{j, i} & =\sum_{k}\left(B_{i k} A_{k j}-A_{i k} B_{k j}\right), \\
B_{i j, k}-B_{i k, j} & =\delta_{i j} C_{k}-\delta_{i k} C_{j}, \\
R_{i j k l}=B_{i k} B_{j l} & -B_{i l} B_{j k}+\delta_{i k} A_{j l}+\delta_{j l} A_{i k}-\delta_{i l} A_{j k}-\delta_{j k} A_{i l},
\end{aligned}
$$


where

$$
R_{i j}:=\sum_{k} R_{i k j k}=-\sum_{k} B_{i k} B_{j k}+(\operatorname{tr} A) \delta_{i j}+(n-2) A_{i j},
$$

$$
\sum_{i} B_{i i}=0, \quad \sum_{i, j}\left(B_{i j}\right)^{2}=\frac{n-1}{n}, \quad \operatorname{tr} A=\sum_{i} A_{i i}=\frac{1}{2 n}\left(1+n^{2} R\right) .
$$

Here $R_{i j k l}$ is the curvature tensor of $g$ defined by the structure equations

$$
d \omega_{i j}-\sum_{k} \omega_{i k} \wedge \omega_{k j}=-\frac{1}{2} \sum_{k, l} R_{i j k l} \omega_{k} \wedge \omega_{l},
$$

and $R=\frac{1}{n(n-1)} \sum_{i, j} R_{i j i j}$ is the normalized Möbius scalar curvature of $x: M^{n} \rightarrow$ $\mathbb{S}^{n+1}$.

The second covariant derivatives of $B_{i j}$ are defined through

$$
\sum_{l} B_{i j, k l} \omega_{l}=d B_{i j, k}+\sum_{l} B_{l j, k} \omega_{l i}+\sum_{l} B_{i l, k} \omega_{l j}+\sum_{l} B_{i j, l} \omega_{l k}
$$

By exterior differentiation of (2-7), we have the Ricci identity

$$
B_{i j, k l}-B_{i j, l k}=\sum_{t} B_{t j} R_{t i k l}+\sum_{t} B_{i t} R_{t j k l} .
$$

From the second of (2-4), we see that the Möbius type operator of $x: M^{n} \rightarrow \mathbb{S}^{n+1}$ takes the form

$$
\Psi=\rho^{-1}(\mathbf{S}-H \mathrm{id})=\sum_{i, j} B_{i j} \omega_{i} E_{j},
$$

which implies that for an umbilic-free hypersurface in $\mathbb{S}^{n+1}$, the number of distinct Möbius principal curvatures is the same as that of its distinct Euclidean principal curvatures.

One can easily show that all coefficients in (2-3) are determined by $\{g, \Psi\}$ and thus we obtain:

Theorem 2.2 [Akivis and Goldberg 1996; 1997; Wang 1998]. Two hypersurfaces $x: M^{n} \rightarrow \mathbb{S}^{n+1}$ and $\tilde{x}: \tilde{M}^{n} \rightarrow \mathbb{S}^{n+1}(n \geq 3)$ are Möbius equivalent if and only if there exists a diffeomorphism $F: M^{n} \rightarrow \tilde{M}^{n}$ that preserves the Möbius metric and the Möbius shape operator.

Recall that an umbilic-free hypersurface $x: M^{n} \rightarrow \mathbb{S}^{n+1}$ is said to have parallel Möbius second fundamental form if $B_{i j, k}=0$ for all $i, j, k$. Hu and Li [2004] established a complete classification for hypersurfaces of $\mathbb{S}^{n+1}$ with parallel Möbius second fundamental form. In particular, we have: 
Theorem 2.3 [Hu and Li 2004]. Let $x: M^{n} \rightarrow \mathbb{S}^{n+1}(n \geq 2)$ be an immersed umbilic-free hypersurface with parallel Möbius second fundamental form and with three distinct principal curvatures. Then $x$ is Möbius equivalent to an open part of the image of $\sigma$ of the warped product embedding

$$
\tilde{x}: \mathbb{S}^{p}(a) \times \mathbb{S}^{q}\left(\sqrt{1-a^{2}}\right) \times \mathbb{R}^{+} \times \mathbb{R}^{n-p-q-1} \rightarrow \mathbb{R}^{n+1},
$$

with $p \geq 1, q \geq 1, p+q \leq n-1$, and $0<a<1$, defined by

$$
\tilde{x}\left(u^{\prime}, u^{\prime \prime}, t, u^{\prime \prime \prime}\right)=\left(t u^{\prime}, t u^{\prime \prime}, t u^{\prime \prime \prime}\right)
$$

with $u^{\prime} \in \mathbb{S}^{p}(a), u^{\prime \prime} \in \mathbb{S}^{q}\left(\sqrt{1-a^{2}}\right), t \in \mathbb{R}^{+}$, and $u^{\prime \prime \prime} \in \mathbb{R}^{n-p-q-1}$. The conformal diffeomorphism $\sigma: \mathbb{R}^{n+1} \rightarrow \mathbb{S}^{n+1} \backslash\{(-1,0, \cdots, 0)\}$ is the inverse of the stereographic projection and is defined by

$$
\sigma(u)=\left(\frac{1-|u|^{2}}{1+|u|^{2}}, \frac{2 u}{1+|u|^{2}}\right), \quad \text { for } u \in \mathbb{R}^{n+1} .
$$

To prove our main theorem, we also need the following partial classification of umbilic-free hypersurfaces in $\mathbb{S}^{n+1}$ with two distinct Blaschke eigenvalues.

Theorem 2.4 [Li and Zhang 2007]. Let $x: M^{n} \rightarrow \mathbb{S}^{n+1}(n \geq 3)$ be an immersed umbilic-free hypersurface with two distinct, constant Blaschke eigenvalues and vanishing Möbius form. If $x$ has three distinct principal Möbius curvatures, then it is locally Möbius equivalent to one of these two families of hypersurfaces in $\mathbb{S}^{n+1}$ :

(i) Minimal hypersurfaces defined by

$$
\begin{aligned}
& \tilde{x}=\left(\tilde{x}_{1}, \tilde{x}_{2}\right): \tilde{M}^{n}=N^{p} \times \mathbb{M}^{n-p}\left(-r^{-2}\right) \rightarrow \mathbb{S}^{n+1}, \\
& \text { where } \quad \tilde{x}_{1}=y_{1} / y_{0}, \quad \tilde{x}_{2}=y_{2} / y_{0},
\end{aligned}
$$

with $y_{0} \in \mathbb{R}^{+}, y_{1} \in \mathbb{R}^{p+2}, y_{2} \in \mathbb{R}^{n-p}, 2 \leq p \leq n-1$, and $r>0$. Also, $y_{1}$ : $N^{p} \rightarrow \mathbb{S}^{p+1}(r) \hookrightarrow \mathbb{R}^{p+2}$ is an immersed umbilic-free minimal hypersurface in the $(p+1)$-dimensional sphere of radius $r$ with constant scalar curvature

$$
\tilde{R}_{1}=\frac{n p(p-1)-(n-1) r^{2}}{n r^{2}} .
$$

$\left(y_{0}, y_{2}\right): \mathbb{\llbracket}^{n-p}\left(-r^{-2}\right) \rightarrow \mathbb{L}^{n-p+1}$ is the standard embedding of the hyperbolic space of sectional curvature $-r^{-2}$ into the $(n-p+1)$-dimensional Lorentz space with $-y_{0}^{2}+y_{2}^{2}=-r^{2}$.

(ii) Nonminimal hypersurfaces defined by

$$
\begin{aligned}
& \tilde{x}=\left(\tilde{x}_{1}, \tilde{x}_{2}\right): \tilde{M}^{n}=N^{p} \times \mathbb{S}^{n-p}(r) \rightarrow \mathbb{S}^{n+1}, \\
& \text { where } \quad \tilde{x}_{1}=y_{1} / y_{0}, \quad \tilde{x}_{2}=y_{2} / y_{0},
\end{aligned}
$$


with $y_{0} \in \mathbb{R}^{+}, y_{1} \in \mathbb{R}^{p+1}, y_{2} \in \mathbb{R}^{n-p+1}, 2 \leq p \leq n-1$, and $r>0$. Also $\left(y_{0}, y_{1}\right)$ : $N^{p} \rightarrow \mathbb{Q}^{p+1}\left(-r^{-2}\right) \hookrightarrow \mathbb{L}^{p+2}$, with $-y_{0}^{2}+y_{1}^{2}=-r^{2}$, is an immersed umbilicfree minimal hypersurface in the $(p+1)$-dimensional hyperbolic space of sectional curvature $-r^{-2}$ and with constant scalar curvature

$$
\tilde{R}_{1}=-\frac{n p(p-1)+(n-1) r^{2}}{n r^{2}} .
$$

$y_{2}: \mathbb{S}^{n-p}(r) \rightarrow \mathbb{R}^{n-p+1}$ is the standard embedding of $(n-p)$-dimensional sphere of radius $r$.

\section{Möbius isoparametric hypersurfaces with $\gamma=3$}

Here, we consider Möbius isoparametric hypersurfaces $x: M^{n} \rightarrow \mathbb{S}^{n+1}$ of any dimension $n \geq 5$ with three distinct principal curvatures with multiplicities $m_{1} \geq$ $m_{2} \geq m_{3}$.

For our choice of the local orthonormal basis $\left\{E_{i}\right\}_{1 \leq i \leq n}$, that $\Psi$ has constant eigenvalues is equivalent to that the matrix $\left(B_{i j}\right)$ has constant eigenvalues. From $\Phi=0$ and (2-9), we see that, for all $i, j$,

$$
\sum_{k}\left(B_{i k} A_{k j}-A_{i k} B_{k j}\right)=0 .
$$

This implies that we can choose $\left\{E_{i}\right\}$ to simultaneously diagonalize $\left(A_{i j}\right)$ and $\left(B_{i j}\right)$. Let us write

$$
\left(B_{i j}\right)=\operatorname{diag}\left(b_{1}, \cdots, b_{n}\right), \quad\left(A_{i j}\right)=\operatorname{diag}\left(A_{1}, \cdots, A_{n}\right),
$$

where the $\left\{b_{i}\right\}$ are constants. By assumption and without loss of generality, we can put

$$
\begin{aligned}
b_{1}=\cdots=b_{m_{1}} & =B_{1}, \\
b_{m_{1}+1}=\cdots=b_{m_{1}+m_{2}} & =B_{2}, \\
b_{m_{1}+m_{2}+1} & =\cdots=b_{n}=B_{3},
\end{aligned}
$$

with distinct $B_{1}, B_{2}$, and $B_{3}$. From (2-13), they satisfy the conditions

$$
m_{1} B_{1}+m_{2} B_{2}+m_{3} B_{3}=0, \quad m_{1} B_{1}^{2}+m_{2} B_{2}^{2}+m_{3} B_{3}^{2}=\frac{n-1}{n} .
$$

In this section, if not stated otherwise, we use the further index conventions

$$
\begin{aligned}
1 & \leq a, b \leq m_{1}, \\
m_{1}+1 & \leq p, q \leq m_{1}+m_{2}, \\
m_{1}+m_{2}+1 & \leq \alpha, \beta \leq m_{1}+m_{2}+m_{3}=n .
\end{aligned}
$$


Applying the condition $\Phi=0$ to (2-8) and (2-10), we see that both $B_{i j, k}$ and $A_{i j, k}$ are totally symmetric. As usual we define

$$
\omega_{i j}=\sum_{k} \Gamma_{k j}^{i} \omega_{k}, \quad \Gamma_{k j}^{i}=-\Gamma_{k i}^{j} .
$$

From this, (2-7), (3-18), and that $\left\{b_{i}\right\}_{1 \leq i \leq n}$ consists of constants, we get

$$
B_{i j, k}=\left(b_{i}-b_{j}\right) \Gamma_{k j}^{i}=\left(b_{j}-b_{k}\right) \Gamma_{i k}^{j}=\left(b_{k}-b_{i}\right) \Gamma_{j i}^{k}, \quad \text { for all } i, j, k .
$$

Thus we see that

(3-21) $B_{i i, j}=B_{i j, i}=B_{a b, j}=B_{p q, j}=B_{\alpha \beta, j}=0, \quad$ for all $i, j, a, b, p, q, \alpha, \beta$,

and the only possible nonzero elements in $\left\{B_{i j, k}\right\}$ are of the form $B_{\alpha a, p}$.

From now on in this section, we assume $B_{i j, k} \not \equiv 0$ and $m_{3}=1$. Then we can prove three lemmas:

Lemma 3.1. The set $\left\{B_{n a, p}\right\}$ has only one nonzero element.

Proof. From the calculation

$$
\begin{aligned}
\sum_{i} B_{a b, p i} \omega_{i} & =d B_{a b, p}+\sum_{i} B_{i b, p} \omega_{i a}+\sum_{i} B_{a i, p} \omega_{i b}+\sum_{i} B_{a b, i} \omega_{i p} \\
& =B_{n b, p} \omega_{n a}+B_{a n, p} \omega_{n b}=\sum_{q}\left(B_{n b, p} \Gamma_{q a}^{n}+B_{n a, p} \Gamma_{q b}^{n}\right) \omega_{q} \\
& =\sum_{q} \frac{B_{n b, p} B_{n a, q}+B_{n a, p} B_{n b, q}}{B_{3}-B_{1}} \omega_{q},
\end{aligned}
$$

we have for all $a, b, p, i$ that

$$
B_{a b, p i}=\frac{B_{n b, p} B_{n a, i}+B_{n a, p} B_{n b, i}}{B_{3}-B_{1}}, \quad B_{a b, p p}=\frac{2 B_{n a, p} B_{n b, p}}{B_{3}-B_{1}} .
$$

On the other hand, from the calculation

$\sum_{i} B_{p p, a i} \omega_{i}=2 \sum_{i} B_{i p, a} \omega_{i p}=2 B_{n p, a} \omega_{n p}=2 \sum_{b} B_{n p, a} \Gamma_{b p}^{n} \omega_{b}=\sum_{b} \frac{2 B_{n a, p} B_{n b, p}}{B_{3}-B_{2}} \omega_{b}$,

we have for all $a, b, p, i$ that

$$
B_{p p, a i}=\frac{2 B_{n a, p} B_{n i, p}}{B_{3}-B_{2}}, \quad B_{p p, a b}=\frac{2 B_{n a, p} B_{n b, p}}{B_{3}-B_{2}} .
$$

For $a \neq b$, using (3-18), (3-19) and (2-11), we obtain from (2-16) that

$$
B_{p p, a b}=B_{p a, p b}=B_{p a, b p}=B_{a b, p p} .
$$

From (3-22)-(3-24), we obtain

$$
B_{n a, p} B_{n b, p}=0, \quad \text { for all } a, b, p \text { with } a \neq b \quad \text { if } m_{1} \geq 2 .
$$


Similarly, we can show

$$
\begin{aligned}
B_{n n, a b} & =\frac{2 \sum_{p} B_{n a, p} B_{n b, p}}{B_{2}-B_{3}}, \quad \text { for all } a, b ; \\
B_{n n, a p} & =0, \quad \text { for all } a, b, p ; \\
B_{p q, a i} & =\frac{B_{n a, p} B_{n i, q}+B_{n a, q} B_{n i, p}}{B_{3}-B_{2}}, \quad \text { for all } a, i, p, q ; \\
B_{n a, p} B_{n a, q} & =0, \quad \text { for all } a, p, q \text { with } p \neq q \text { if } m_{2} \geq 2 .
\end{aligned}
$$

From (3-22), (3-28) and that

$$
B_{a b, p q}=B_{p q, a b}, \quad \text { if } a \neq b \text { or } p \neq q,
$$

we obtain

(3-30) $\quad B_{n a, p} B_{n b, q}+B_{n a, q} B_{n b, p}=0, \quad$ for all $a, b, p, q$ with $(a, p) \neq(b, q)$.

As $B_{i j, k} \not \equiv 0$, we can take indices $\bar{a}$ and $\bar{p}$ to satisfy

$$
B_{n \bar{a}, \bar{p}} \neq 0, \quad \bar{a} \in\left\{1, \cdots, m_{1}\right\}, \bar{p} \in\left\{m_{1}+1, \cdots, m_{1}+m_{2}\right\} .
$$

Then from (3-25) and (3-29), we have

$$
B_{n b, \bar{p}}=0, \quad \text { for all } b \neq \bar{a} \text { if } m_{1} \geq 2, \quad B_{n \bar{a}, q}=0, \quad \text { for all } q \neq \bar{p} \text { if } m_{2} \geq 2,
$$

and this, together with (3-30), gives $B_{n \bar{a}, \bar{p}} B_{n b, q}=0$ for all $(b, q) \neq(\bar{a}, \bar{p})$. Therefore, we have $B_{n b, q}=0$ for all $(b, q) \neq(\bar{a}, \bar{p})$.

Lemma 3.2. For the indices $\bar{a}, \bar{p}$ in Lemma 3.1, the $B_{n \bar{a}, \bar{p}}$ are constant.

Proof. From Lemma 3.1 and (2-15), we have $d B_{n \bar{a}, \bar{p}}=\sum_{i} B_{n \bar{a}, \bar{p} i} \omega_{i}$. Seeing that the four indices in $B_{n \bar{a}, \bar{p} i}$ are totally symmetric, using (3-22), (3-27) and (3-28), we get for all $b, q$ that

$$
B_{n \bar{a}, \bar{p} b}=B_{\bar{a} b, \bar{p} n}=0, \quad B_{n \bar{a}, \bar{p} q}=B_{\bar{p} q, \bar{a} n}=0, \quad B_{n \bar{a}, \bar{p} n}=B_{n n, \bar{a} \bar{p}}=0 .
$$

Lemma 3.3. We have these results for the sectional curvature:

$$
\begin{array}{rlrl}
R_{\text {apap }}=0, & \text { for all }(a, p) \neq(\bar{a}, \bar{p}) ; & R_{\bar{a} \bar{p} \bar{a} \bar{p}} & =\frac{2 B_{n \bar{a}, \bar{p}}^{2}}{\left(B_{1}-B_{3}\right)\left(B_{2}-B_{3}\right)} ; \\
R_{\text {nana }}=0, & \text { for all } a \neq \bar{a} ; & R_{n \bar{a} n \bar{a}}=\frac{2 B_{n \bar{a}, \bar{p}}^{2}}{\left(B_{1}-B_{2}\right)\left(B_{3}-B_{2}\right)} ; \\
R_{n p n p}=0, \text { for all } p \neq \bar{p} ; & R_{n \bar{p} n \bar{p}}=\frac{2 B_{n \bar{a}, \bar{p}}^{2}}{\left(B_{2}-B_{1}\right)\left(B_{3}-B_{1}\right)} .
\end{array}
$$


Proof. Now we have the facts

$$
\begin{aligned}
\omega_{a p}=0, & \text { if }(a, p) \neq(\bar{a}, \bar{p}) ; & \omega_{\bar{a} \bar{p}} & =\frac{B_{n \bar{a}, \bar{p}}}{B_{1}-B_{2}} \omega_{n} ; \\
\omega_{n a}=0, & \text { if } a \neq \bar{a} ; & \omega_{n \bar{a}} & =\frac{B_{n \bar{a}, \bar{p}}}{B_{3}-B_{1}} \omega_{\bar{p}} ; \\
\omega_{n p}=0, & \text { if } p \neq \bar{p} ; & \omega_{n \bar{p}} & =\frac{B_{n \bar{a}, \bar{p}}}{B_{3}-B_{2}} \omega_{\bar{a}} .
\end{aligned}
$$

According to this, (2-14), and $B_{n \bar{a}, \bar{p}}$ being constant, we have, for all $a \neq \bar{a}$ and $p \neq \bar{p}$,

$-\frac{1}{2} \sum_{i, j} R_{a p i j} \omega_{i} \wedge \omega_{j}=d \omega_{a p}-\sum_{i} \omega_{a i} \wedge \omega_{i p}=0$,

$-\frac{1}{2} \sum_{i, j} R_{a \bar{p} i j} \omega_{i} \wedge \omega_{j}=d \omega_{a \bar{p}}-\sum_{i} \omega_{a i} \wedge \omega_{i \bar{p}}=-\omega_{a \bar{a}} \wedge \omega_{\bar{a} \bar{p}}=-\frac{B_{n \bar{a}}, \bar{p}}{B_{1}-B_{2}} \omega_{a \bar{a}} \wedge \omega_{n}$,

$-\frac{1}{2} \sum_{i, j} R_{\bar{a} p i j} \omega_{i} \wedge \omega_{j}=d \omega_{\bar{a} p}-\sum_{i} \omega_{\bar{a} i} \wedge \omega_{i p}=-\omega_{\bar{a} \bar{p}} \wedge \omega_{\bar{p} p}=-\frac{B_{n \bar{a}, \bar{p}}}{B_{1}-B_{2}} \omega_{n} \wedge \omega_{\bar{p} p}$,

$-\frac{1}{2} \sum_{i, j} R_{n a i j} \omega_{i} \wedge \omega_{j}=d \omega_{n a}-\sum_{i} \omega_{n i} \wedge \omega_{i a}=-\omega_{n \bar{a}} \wedge \omega_{\bar{a} a}=-\frac{B_{n \bar{a}}, \bar{p}}{B_{3}-B_{1}} \omega_{\bar{p}} \wedge \omega_{\bar{a} a}$,

$-\frac{1}{2} \sum_{i, j} R_{n p i j} \omega_{i} \wedge \omega_{j}=d \omega_{n p}-\sum_{i} \omega_{n i} \wedge \omega_{i p}=-\omega_{n \bar{p}} \wedge \omega_{\bar{p} p}=-\frac{B_{n \bar{a}}, \bar{p}}{B_{3}-B_{2}} \omega_{\bar{a}} \wedge \omega_{\bar{p} p}$,

$-\frac{1}{2} \sum_{i, j} R_{n \bar{a} i j} \omega_{i} \wedge \omega_{j}=d \omega_{n \bar{a}}-\sum_{i} \omega_{n i} \wedge \omega_{i \bar{a}}=d\left(\Gamma_{\bar{p} \bar{a}}^{n} \omega_{\bar{p}}\right)-\omega_{n \bar{p}} \wedge \omega_{\bar{p} \bar{a}}$

$$
\begin{array}{r}
=\Gamma_{\bar{p} \bar{a}}^{n} \sum_{i} \omega_{\bar{p} i} \wedge \omega_{i}-\omega_{n \bar{p}} \wedge \omega_{\bar{p} \bar{a}}=\Gamma_{\bar{p} \bar{a}}^{n}\left(\omega_{\bar{p} \bar{a}} \wedge \omega_{\bar{a}}+\omega_{\bar{p} n} \wedge \omega_{n}\right)-\omega_{n \bar{p}} \wedge \omega_{\bar{p} \bar{a}} \\
=-\frac{2 B_{n \bar{a}, \bar{p}}^{2}}{\left(B_{1}-B_{2}\right)\left(B_{3}-B_{2}\right)} \omega_{n} \wedge \omega_{\bar{a}} .
\end{array}
$$

Similarly,

$$
\begin{aligned}
& -\frac{1}{2} \sum_{i, j} R_{n \bar{p} i j} \omega_{i} \wedge \omega_{j}=d \omega_{n \bar{p}}-\sum_{i} \omega_{n i} \wedge \omega_{i \bar{p}}=-\frac{2 B_{n \bar{a}, \bar{p}}^{2}}{\left(B_{2}-B_{1}\right)\left(B_{3}-B_{1}\right)} \omega_{n} \wedge \omega_{\bar{p}}, \\
& -\frac{1}{2} \sum_{i, j} R_{\bar{a} \bar{p} i j} \omega_{i} \wedge \omega_{j}=d \omega_{\bar{a} \bar{p}}-\sum_{i} \omega_{\bar{a} i} \wedge \omega_{i \bar{p}}=-\frac{2 B_{n \bar{a}, \bar{p}}^{2}}{\left(B_{1}-B_{3}\right)\left(B_{2}-B_{3}\right)} \omega_{\bar{a}} \wedge \omega_{\bar{p}}
\end{aligned}
$$

From the equations above, we come to the conclusion immediately.

Now, we are ready to prove:

Proposition 3.4. Let $x: M^{n} \rightarrow \mathbb{S}^{n+1}(n \geq 5)$ be a Möbius isoparametric hypersurface with three distinct Möbius principal curvatures of multiplicities $m_{1} \geq$ $m_{2} \geq m_{3}$. If the Möbius second fundamental form is not parallel and $m_{3}=1$, then 
$m_{2}=1$ and the Möbius principal curvatures are $B_{1}=0$ with multiplicity $n-2$ and $B_{2}=-B_{3}= \pm \sqrt{(n-1) / 2 n}$.

Proof. From Lemma 3.3 and the Gauss Equation (2-11), we obtain the equations

$$
\begin{array}{rlrl}
R_{a \bar{p} a \bar{p}}=B_{1} B_{2}+A_{a}+A_{\bar{p}}=0, & a \neq \bar{a}, \\
R_{\bar{a} p \bar{a} p}=B_{1} B_{2}+A_{\bar{a}}+A_{p}=0, & p \neq \bar{p} \\
R_{\text {apap }}=B_{1} B_{2}+A_{a}+A_{p}=0, & a \neq \bar{a}, \quad p \neq \bar{p}, \\
R_{\text {nana }}=B_{1} B_{3}+A_{a}+A_{n}=0, & a \neq \bar{a}, \\
R_{n p n p}=B_{2} B_{3}+A_{p}+A_{n}=0, & p \neq \bar{p}, \\
R_{n \bar{a} n \bar{a}}=B_{1} B_{3}+A_{\bar{a}}+A_{n}=\frac{2 B_{n \bar{a}, \bar{p}}^{2}}{\left(B_{1}-B_{2}\right)\left(B_{3}-B_{2}\right)}, \\
R_{n \bar{p} n \bar{p}}=B_{2} B_{3}+A_{\bar{p}}+A_{n}=\frac{2 B_{n \bar{a}, \bar{p}}^{2}}{\left(B_{2}-B_{1}\right)\left(B_{3}-B_{1}\right)}, \\
R_{\bar{a} \bar{p} \bar{a} \bar{p}}=B_{1} B_{2}+A_{\bar{a}}+A_{\bar{p}}=\frac{2 B_{n \bar{a}, \bar{p}}^{2}}{\left(B_{1}-B_{3}\right)\left(B_{2}-B_{3}\right)} .
\end{array}
$$

If $m_{2} \geq 2$, then we can form (3-31) $+(3-36)-(3-34)-(3-38)$ and (3-32) $+(3-37)-$ $(3-35)-(3-38)$, which give $\left(B_{2}-B_{3}\right)\left(2 B_{1}-B_{2}-B_{3}\right)=0$ and $\left(B_{1}-B_{3}\right)\left(2 B_{2}-\right.$ $\left.B_{1}-B_{3}\right)=0$, respectively. Therefore we have

$$
2 B_{1}-B_{2}-B_{3}=2 B_{2}-B_{1}-B_{3}=0,
$$

which implies $B_{1}=B_{2}=B_{3}$. This contradiction thus means we should have $m_{2}=1$.

Now for $m_{2}=1$, we form (3-31) + (3-36) - (3-34) - (3-38) once again to obtain $2 B_{1}=B_{2}+B_{3}$. On the other hand, the first equation of (3-20) now reads $(n-2) B_{1}+B_{2}+B_{3}=0$. These imply that $B_{1}=B_{2}+B_{3}=0$. Now applying the second equation of (3-20), we obtain

$$
B_{2}=-B_{3}= \pm \sqrt{\frac{n-1}{2 n}} .
$$

In the rest of this section, we assume $B_{i j, k} \not \equiv 0$ and $m_{2}=m_{3}=1$. Without loss of generality, we assume that

$$
B_{1}=0, \quad B_{2}=\sqrt{\frac{n-1}{2 n}}, \quad B_{3}=-\sqrt{\frac{n-1}{2 n}} \quad B_{\bar{a} \bar{p}, n} \neq 0,
$$

where, for simplicity, we use the notation $\bar{p}=n-1, \bar{a}=n-2$. 
Lemma 3.5. With the assumptions above, we have for all $a \neq \bar{a}$ that

$$
\begin{aligned}
\omega_{a \bar{a}} & =\omega_{a \bar{p}}=\omega_{a n}=0, & R_{a \bar{p} a \bar{p}} & =R_{\text {anan }}=0, \\
\omega_{\bar{a} \bar{p}} & =\frac{B_{n \bar{a}, \bar{p}}}{B_{1}-B_{2}} \omega_{n}, & R_{\bar{a} \bar{p} \bar{a} \bar{p}} & =\frac{2 B_{n \bar{a}, \bar{p}}^{2}}{\left(B_{1}-B_{3}\right)\left(B_{2}-B_{3}\right)}, \\
\omega_{\bar{a} n} & =\frac{B_{n \bar{a}, \bar{p}}}{B_{1}-B_{3}} \omega_{\bar{p}}, & R_{\bar{a} n \bar{a} n} & =\frac{2 B_{n \bar{a}, \bar{p}}^{2}}{\left(B_{1}-B_{2}\right)\left(B_{3}-B_{2}\right)}, \\
\omega_{\bar{p} n} & =\frac{B_{n \bar{a}, \bar{p}}}{B_{2}-B_{3}} \omega_{\bar{a}}, & R_{\bar{p} n \bar{p} n} & =\frac{2 B_{n \bar{a}, \bar{p}}^{2}}{\left(B_{2}-B_{1}\right)\left(B_{3}-B_{1}\right)} .
\end{aligned}
$$

Proof. These are direct consequences of (3-21), Lemma 3.3, and the equations that finish its proof. To make it clear that $\omega_{a \bar{a}}=0$ for all $a \neq \bar{a}$, we note

$$
\omega_{a \bar{a}} \wedge \omega_{\bar{p}}=\omega_{a \bar{a}} \wedge \omega_{n}=0, \quad \text { for all } a \neq \bar{a},
$$

which are also implied by the second and fourth equations in the second group in the proof of Lemma 3.3.

Lemma 3.6. In our situation, we have for all $a \neq \bar{a}$

$$
\begin{gathered}
A_{a}=-A_{\bar{a}}=-A_{\bar{p}}=-A_{n}=-\frac{n-1}{12 n}, \quad B_{n \bar{a}, \bar{p}}= \pm \frac{n-1}{6 n} \sqrt{3}, \\
R_{a \bar{a} a \bar{a}}=0, \quad R_{\bar{a} \bar{p} \bar{a} \bar{p}}=\frac{n-1}{6 n}, \quad R_{\bar{a} n \bar{a} n}=\frac{n-1}{6 n}, \quad R_{\bar{p} n \bar{p} n=-\frac{n-1}{3 n} .}
\end{gathered}
$$

Furthermore, also for $a \neq \bar{a}$, the first structure equations can be written as

$$
\begin{array}{ll}
d \omega_{a}=\sum_{b \neq \bar{a}} \omega_{a b} \wedge \omega_{b}, & d \omega_{\bar{a}}= \pm \sqrt{\frac{2(n-1)}{3 n}} \omega_{\bar{p}} \wedge \omega_{n}, \\
d \omega_{\bar{p}}=\mp \sqrt{\frac{n-1}{6 n}} \omega_{\bar{a}} \wedge \omega_{n}, & d \omega_{n}= \pm \sqrt{\frac{n-1}{6 n}} \omega_{\bar{a}} \wedge \omega_{\bar{p}} .
\end{array}
$$

Proof. Assuming always that $a \neq \bar{a}$, from (3-39), Lemma 3.5, and the Gauss Equation (2-11), we now have

$$
\begin{aligned}
& R_{a \bar{p} a \bar{p}}=A_{a}+A_{\bar{p}}=R_{\text {anan }}=A_{a}+A_{n}=0, \\
& R_{\bar{a} \bar{p} \bar{a} \bar{p}}=A_{\bar{a}}+A_{\bar{p}}=R_{\bar{a} n \bar{a} n}=A_{\bar{a}}+A_{n}=\frac{2 n}{n-1} B_{n \bar{a}, \bar{p}}^{2}, \\
& R_{\bar{p} n \bar{p} n}=-\frac{n-1}{2 n}+A_{\bar{p}}+A_{n}=-\frac{4 n}{n-1} B_{n \bar{a}, \bar{p}}^{2} .
\end{aligned}
$$


From these, we obtain

$$
\begin{aligned}
A_{a}=-A_{\bar{p}}=-A_{n} & =-\frac{n-1}{4 n}+\frac{2 n}{n-1} B_{n \bar{a}, \bar{p}}^{2}, \\
A_{\bar{a}} & =-\frac{n-1}{4 n}+\frac{4 n}{n-1} B_{n \bar{a}, \bar{p}}^{2} .
\end{aligned}
$$

On the other hand, because $\omega_{a \bar{a}}=\omega_{a \bar{p}}=\omega_{a n}=0$, we obtain from (2-14) that $R_{a \bar{a} a \bar{a}}=0$, According to $R_{a \bar{a} a \bar{a}}=B_{1}^{2}+A_{a}+A_{\bar{a}}$, we find $A_{a}=-A_{\bar{a}}$. Putting this into the above, we find $B_{n \bar{a}, \bar{p}}^{2}=((n-1) / n)^{2} / 12$. Then our conclusion follows immediately from Lemma 3.5.

Lemma 3.6 shows that, in this case, the Blaschke tensor has exactly two distinct constant eigenvalues. Therefore, as an application of Theorem 2.4, we have proved:

Theorem 3.7. Let $x: M^{n} \rightarrow \mathbb{S}^{n+1}(n \geq 5)$ be a Möbius isoparametric hypersurface with nonparallel Möbius second fundamental form and three distinct Möbius principal curvatures, one of which is simple. Then locally $x$ can only be Möbius equivalent to one of these two families of hypersurfaces in $\mathbb{S}^{n+1}$ :

$\left(\mathfrak{C}_{1}\right)$ Minimal hypersurfaces defined by

$$
\begin{aligned}
& \tilde{x}=\left(\tilde{x}_{1}, \tilde{x}_{2}\right): \tilde{M}^{n}=N^{p} \times \mathbb{T}^{n-p}\left(-r^{-2}\right) \rightarrow \mathbb{S}^{n+1}, \\
& \text { where } \tilde{x}_{1}=y_{1} / y_{0}, \quad \tilde{x}_{2}=y_{2} / y_{0},
\end{aligned}
$$

with $y_{0} \in \mathbb{R}^{+}, y_{1} \in \mathbb{R}^{p+2}, y_{2} \in \mathbb{R}^{n-p}, 2 \leq p \leq n-1$, and $r>0$. Also, $y_{1}$ : $N^{p} \rightarrow \mathbb{S}^{p+1}(r) \hookrightarrow \mathbb{R}^{p+2}$ is an immersed umbilic-free minimal hypersurface in the $(p+1)$-dimensional sphere of radius $r$ and with constant scalar curvature

$$
\tilde{R}_{1}=\frac{n p(p-1)-(n-1) r^{2}}{n r^{2}} .
$$

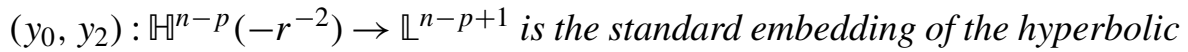
space of sectional curvature $-r^{-2}$ into the $(n-p+1)$-dimensional Lorentz space with $-y_{0}^{2}+y_{2}^{2}=-r^{2}$.

$\left(\mathfrak{C}_{2}\right)$ Nonminimal hypersurfaces defined by

$$
\begin{aligned}
& \tilde{x}=\left(\tilde{x}_{1}, \tilde{x}_{2}\right): \tilde{M}^{n}=N^{p} \times \mathbb{S}^{n-p}(r) \rightarrow \mathbb{S}^{n+1}, \\
& \text { where } \quad \tilde{x}_{1}=y_{1} / y_{0}, \quad \tilde{x}_{2}=y_{2} / y_{0},
\end{aligned}
$$

with $y_{0} \in \mathbb{R}^{+}, y_{1} \in \mathbb{R}^{p+1}, y_{2} \in \mathbb{R}^{n-p+1}, 2 \leq p \leq n-1$, and $r>0$. Also $\left(y_{0}, y_{1}\right)$ : $N^{p} \rightarrow \mathbb{R}^{p+1}\left(-r^{-2}\right) \hookrightarrow \mathbb{L}^{p+2}$, with $-y_{0}^{2}+y_{1}^{2}=-r^{2}$, is an immersed umbilicfree minimal hypersurface in the $(p+1)$-dimensional hyperbolic space of 
sectional curvature $-r^{-2}$ and with constant scalar curvature

$$
\tilde{R}_{1}=-\frac{n p(p-1)+(n-1) r^{2}}{n r^{2}} .
$$

$y_{2}: \mathbb{S}^{n-p}(r) \rightarrow \mathbb{R}^{n-p+1}$ is the standard embedding of $(n-p)$-dimensional sphere of radius $r$.

To answer which of the hypersurfaces in $\mathfrak{C}_{1}$ and $\mathfrak{C}_{2}$ are Möbius isoparametric, we need to calculate their Möbius invariants, and we do this next.

\section{Möbius invariants of hypersurfaces in $\mathfrak{C}_{1}$ and $\mathfrak{C}_{2}$}

Keeping in mind that some of the hypersurfaces in $\mathfrak{C}_{1}$ and $\mathfrak{C}_{2}$ might be not Möbius isoparametric, we will sort them out by direct calculation.

Example 4.1. Calculation for hypersurfaces in $\mathfrak{C}_{1}$. Compare with [Li and Zhang 2007].

For $\tilde{x}=\left(\tilde{x}_{1}, \tilde{x}_{2}\right)$ as defined under $\mathfrak{C}_{1}$ in Theorem 3.7, we have

$$
d \tilde{x}=-\frac{d y_{0}}{y_{0}^{2}}\left(y_{1}, y_{2}\right)+\frac{1}{y_{0}}\left(d y_{1}, d y_{2}\right),
$$

and then its Euclidean induced metric is given by

$$
\tilde{I}=d \tilde{x} \cdot d \tilde{x}=\left.y_{0}^{-2}\left(-d y_{0}^{2}+d y_{1}^{2}+d y_{2}^{2}\right)\right|_{\tilde{M}^{n}} .
$$

Let $\xi_{1}$ be the unit normal vector field of $y_{1}: N^{p} \rightarrow \mathbb{S}^{p+1}(r) \hookrightarrow \mathbb{R}^{p+2}$. Then $\xi=\left(\xi_{1}, 0\right) \in \mathbb{R}^{n+2}$ is a unit normal vector field of $\tilde{x}$. Consequently, by (4-43), the (Euclidean) second fundamental form $\tilde{h}$ of $\tilde{x}$ is related to the (Euclidean) second fundamental form $\tilde{h}^{*}$ of $y_{1}$ by

$$
\tilde{h}=-d \xi \cdot d \tilde{x}=-y_{0}^{-1}\left(d \xi_{1} \cdot d y_{1}\right)=y_{0}^{-1} \tilde{h}^{*} .
$$

Let $\left\{\tilde{E}_{i}\right\}_{1 \leq i \leq p}$ and $\left\{\tilde{E}_{i}\right\}_{p+1 \leq i \leq n}$ be the local orthonormal bases on $\left(N^{p}, d y_{1}^{2}\right)$ and $\mathbb{M}^{n-p}\left(-r^{-2}\right)$, respectively. Then $\left\{\tilde{E}_{i}\right\}_{1 \leq i \leq n}$ form a local orthonormal basis on $\tilde{M}^{n}$ with respect to the metric $\left.\left(-d y_{0}^{2}+d y_{1}^{2}+d y_{2}^{2}\right)\right|_{\tilde{M}^{n}}=y_{0}^{2} \tilde{I}$.

Put $\tilde{e}_{i}=y_{0} \tilde{E}_{i}, 1 \leq i \leq n$. Then $\left\{\tilde{e}_{i}\right\}_{1 \leq i \leq n}$ is a local orthonormal basis on $\tilde{M}^{n}$ with respect to the metric $\tilde{I}$. Thus

$$
\begin{aligned}
\tilde{h}_{i j} & =\tilde{h}\left(\tilde{e}_{i}, \tilde{e}_{j}\right)=y_{0}^{2} \tilde{h}\left(\tilde{E}_{i}, \tilde{E}_{j}\right) & & \\
& =y_{0} \tilde{h}^{*}\left(\tilde{E}_{i}, \tilde{E}_{j}\right)=y_{0} \tilde{h}_{i j}^{*} & & \text { if } 1 \leq i, j \leq p, \\
\tilde{h}_{i j} & =0 & & \text { if } i>p \text { or } j>p .
\end{aligned}
$$


From this and the minimality of $y_{1}$, we see that $\tilde{x}: \tilde{M}^{n} \rightarrow \mathbb{S}^{n+1}$ is also minimal, that is, $\tilde{H}=0$. Therefore, by definition, the Möbius factor $\tilde{\rho}$ of $\tilde{x}$ is determined by

$$
\tilde{\rho}^{2}=\frac{n}{n-1}\left(\sum_{i, j=1}^{n} \tilde{h}_{i j}^{2}-n \tilde{H}^{2}\right)=\frac{n}{n-1} y_{0}^{2} \sum_{i, j=1}^{p}\left(\tilde{h}_{i j}^{*}\right)^{2}=y_{0}^{2},
$$

where in the last equality, we use that $\sum_{i, j=1}^{p}\left(\tilde{h}_{i j}^{*}\right)^{2}=(n-1) / n$, which is implied by (3-41) and the Gauss equation of $y_{1}$. Hence, the Möbius position vector of $\tilde{x}$ is $\tilde{Y}=\tilde{\rho}(1, \tilde{x})=\left(y_{0}, y_{1}, y_{2}\right) \in \mathbb{L}^{n+3}$ and the Möbius metric of $\tilde{x}$ is

$$
\tilde{g}=\langle d \tilde{Y}, d \tilde{Y}\rangle=\left.\left(-d y_{0}^{2}+d y_{1}^{2}+d y_{2}^{2}\right)\right|_{\tilde{M}^{n}}=y_{0}^{2} \tilde{I} .
$$

Therefore, $\left\{\tilde{E}_{i}\right\}_{1 \leq i \leq n}$ is in fact a local orthonormal basis of the Möbius metric $\tilde{g}$. Furthermore, the Möbius second fundamental form of $\tilde{x}$ is

$$
\tilde{\mathbf{B}}=\tilde{\rho}^{-1} \sum_{i, j=1}^{n}\left(\tilde{h}_{i j}-\tilde{H} \delta_{i j}\right) \tilde{\omega}_{i} \tilde{\omega}_{j}=\sum_{i, j=1}^{p} \tilde{h}_{i j}^{*} \tilde{\omega}_{i} \tilde{\omega}_{j}
$$

where $\left\{\tilde{\omega}_{i}\right\}_{1 \leq i \leq n}$ is the dual basis of $\left\{\tilde{E}_{i}\right\}_{1 \leq i \leq n}$ on $\tilde{M}^{n}$. Note that (4-46) is equivalent to

$$
\tilde{B}_{i j}=\tilde{h}_{i j}^{*}, \quad \text { for } 1 \leq i, j \leq p ; \quad \tilde{B}_{i j}=0, \quad \text { for } i>p \text { or } j>p .
$$

Since (4-45) shows that $\left(\tilde{M}^{n}, \tilde{g}\right)$ is the Riemannian direct product

$$
\left(\tilde{M}^{n}, \tilde{g}\right)=\left(N^{p}, d y_{1}^{2}\right) \times \mathbb{H}^{n-p}\left(-r^{-2}\right),
$$

we can use the Gauss equation to write down the Ricci tensor of $\tilde{g}$ with respect to $\left\{\tilde{E}_{i}\right\}_{1 \leq i \leq n}$ as:

$$
\tilde{R}_{i j}= \begin{cases}\frac{p-1}{r^{2}} \delta_{i j}-\sum_{k=1}^{p} \tilde{h}_{i k}^{*} \tilde{h}_{k j}^{*}, & \text { if } 1 \leq i, j \leq p ; \\ -\frac{n-p-1}{r^{2}} \delta_{i j}, & \text { if } p+1 \leq i, j \leq n \\ 0, & \text { for all other cases }\end{cases}
$$

which implies that the normalized scalar curvature $\tilde{R}$ of $\tilde{g}$ satisfies

$$
\begin{aligned}
n(n-1) \tilde{R} & =\frac{p(p-1)-(n-p)(n-p-1)}{r^{2}}-\sum_{i, j=1}^{p}\left(\tilde{h}_{i j}^{*}\right)^{2} \\
& =\frac{p(p-1)-(n-p)(n-p-1)}{r^{2}}-\frac{n-1}{n} .
\end{aligned}
$$


Thus

$$
\frac{1}{2 n}\left(1+n^{2} \tilde{R}\right)=\frac{p(p-1)-(n-p)(n-p-1)}{2(n-1) r^{2}} .
$$

From (2-12), (2-13), and (4-47)-(4-50), it follows easily that the Blaschke tensor of $\tilde{x}$ is given by $\tilde{\mathbf{A}}=\sum_{i, j=1}^{n} \tilde{A}_{i j} \tilde{\omega}_{i} \tilde{\omega}_{j}$, where the $\tilde{A}_{i j}$ form a diagonal matrix with entries $\tilde{A}_{i i}=1 /\left(2 r^{2}\right)$ if $1 \leq i \leq p, \tilde{A}_{i i}=-1 /\left(2 r^{2}\right)$ if $p+1 \leq i \leq n$, and 0 elsewhere.

For the Möbius form $\tilde{\Phi}=\sum_{i=1}^{n} \tilde{C}_{i} \tilde{\omega}_{i}$ of $\tilde{x}$, from (2-4), (4-44) and that $\tilde{H}=$ $0, \tilde{\rho}=y_{0}$, we see, for $1 \leq i \leq n$, that

$$
\tilde{C}_{i}=-\tilde{\rho}^{-2}\left(\tilde{e}_{i}(\tilde{H})+\sum_{j=1}^{n}\left(\tilde{h}_{i j}-\tilde{H} \delta_{i j}\right) \tilde{e}_{j}(\log \tilde{\rho})\right)=-y_{0}^{-1} \sum_{j=1}^{p} \tilde{h}_{i j}^{*} \tilde{e}_{j}\left(\log y_{0}\right)=0
$$

Therefore, we have $\tilde{\Phi}=0$. To summarize the above calculation, we present:

Proposition 4.2. A hypersurface $\tilde{x}$ in $\mathfrak{C}_{1}$ is Möbius isoparametric if and only if it satisfies:

(i) $p=3$;

(ii) $r=\sqrt{6 n /(n-1)}$;

(iii) $y_{1}: N^{3} \rightarrow \mathbb{S}^{4}(\sqrt{6 n /(n-1)})$ is a minimal isoparametric hypersurface with vanishing scalar curvature; moreover, it has three distinct principal curvatures with values $\pm \sqrt{(n-1) /(2 n)}, 0$.

Proof. From (3-40) and the expression for $\tilde{\mathbf{A}}$, we see that $p=3$ and $r=\sqrt{6 n /(n-1)}$. From (3-41) we find that $y_{1}: N^{3} \rightarrow \mathbb{S}^{4}(r)$ has vanishing scalar curvature. From (4-46), we know that all the nonzero Möbius principal curvatures of $\tilde{x}$ are equal to the nonzero Euclidean principal curvatures of $y_{1}$. From (3-39), we then deduce that the principal curvatures of $y_{1}$ are exactly $\pm \sqrt{(n-1) /(2 n)}, 0$.

Remark 4.3. According to E. Cartan [1939], minimal isoparametric hypersurfaces in $\mathbb{S}^{4}(r), r=\sqrt{6 n /(n-1)}$, with three distinct principal curvatures do exist, and they are unique. More precisely, the hypersurface is the tube of constant radius over the standard Veronese embedding of $\mathbb{R} P^{2}$ into $\mathbb{S}^{4}(r)$ with principal curvatures $\pm \sqrt{(n-1) /(2 n)}, 0$.

Example 4.4. Calculation for hypersurfaces in $\mathfrak{C}_{2}$. Compare with [Li and Zhang 2007].

For $\tilde{x}=\left(\tilde{x}_{1}, \tilde{x}_{2}\right)$ as defined under $\mathfrak{C}_{2}$ in Theorem 3.7, we have

$$
d \tilde{x}=-\frac{d y_{0}}{y_{0}^{2}}\left(y_{1}, y_{2}\right)+\frac{1}{y_{0}}\left(d y_{1}, d y_{2}\right),
$$


and then its Euclidean induced metric is

$$
\tilde{I}=d \tilde{x} \cdot d \tilde{x}=\left.y_{0}^{-2}\left(-d y_{0}^{2}+d y_{1}^{2}+d y_{2}^{2}\right)\right|_{\tilde{M}^{n}} .
$$

Let $\left(\xi_{0}, \xi_{1}\right)$ be the unit normal vector field of

$$
\tilde{y}:=\left(y_{0}, y_{1}\right): N^{p} \rightarrow \mathbb{H}^{p+1}\left(-r^{-2}\right) \hookrightarrow \mathbb{Q}^{p+2},
$$

where $\xi_{0} \in \mathbb{R}^{+}, \xi_{1} \in \mathbb{R}^{p+1}$. Then we can easily verify that

$$
\xi=\left(\xi_{1}, 0\right)-\xi_{0} \tilde{x} \in \mathbb{R}^{n+2}
$$

is a unit normal vector field of $\tilde{x}$, and the Euclidean second fundamental form $\tilde{h}$ of $\tilde{x}$ is given by

$$
\begin{aligned}
\tilde{h} & =-d \xi \cdot d \tilde{x}=\xi_{0} d \tilde{x} \cdot d \tilde{x}-\left(d \xi_{1}, 0\right) \cdot d \tilde{x}=-y_{0}^{-1}\left(-d \xi_{0} d y_{0}+d \xi_{1} \cdot d y_{1}\right)+\xi_{0} \tilde{I} \\
& =-y_{0}^{-1}\left\langle d\left(\xi_{0}, \xi_{1}\right), d\left(y_{0}, y_{1}\right)\right\rangle+\xi_{0} \tilde{I}=y_{0}^{-1} \tilde{h}^{*}+\xi_{0} \tilde{I},
\end{aligned}
$$

where $\tilde{h}^{*}$ denotes the Euclidean second fundamental form of $\tilde{y}: N^{p} \rightarrow \mathbb{M}^{p+1}\left(-r^{-2}\right)$ and, in the third equality, we have used $d \xi_{1} \cdot y_{1}=y_{0} d \xi_{0}$ which is implied by

$$
-\xi_{0} y_{0}+\xi_{1} \cdot y_{1}=-\xi_{0} d y_{0}+\xi_{1} \cdot d y_{1}=0 .
$$

Let $\left\{\tilde{E}_{i}\right\}_{1 \leq i \leq p}$ and $\left\{\tilde{E}_{i}\right\}_{p+1 \leq i \leq n}$ be the local orthonormal bases on $\left(N^{p}, d \tilde{y}^{2}\right)$ and $\mathbb{S}^{n-p}(r)$, respectively. Then $\left\{\tilde{E}_{i}\right\}_{1 \leq i \leq n}$ form a local orthonormal basis on $\tilde{M}^{n}$ with respect to the metric $\left.\left(-d y_{0}^{2}+d y_{1}^{2}+d y_{2}^{2}\right)\right|_{\tilde{M}^{n}}=y_{0}^{2} \tilde{I}$.

Put $\tilde{e}_{i}=y_{0} \tilde{E}_{i}, 1 \leq i \leq n$. Then $\left\{\tilde{e}_{i}\right\}_{1 \leq i \leq n}$ is a local orthonormal basis on $\tilde{M}^{n}$ with respect to the metric $\tilde{I}$. Thus, we have, for $1 \leq i, j \leq p$,

$$
\tilde{h}_{i j}=\tilde{h}\left(\tilde{e}_{i}, \tilde{e}_{j}\right)=y_{0}^{2} \tilde{h}\left(\tilde{E}_{i}, \tilde{E}_{j}\right)=y_{0} \tilde{h}^{*}\left(\tilde{E}_{i}, \tilde{E}_{j}\right)+\xi_{0} y_{0}^{2} \tilde{I}\left(\tilde{E}_{i}, \tilde{E}_{j}\right)=y_{0} \tilde{h}_{i j}^{*}+\xi_{0} \delta_{i j}
$$

and, for $i>p$ or $j>p$,

$$
\tilde{h}_{i j}=y_{0}^{2} \tilde{h}\left(\tilde{E}_{i}, \tilde{E}_{j}\right)=y_{0} \tilde{h}^{*}\left(\tilde{E}_{i}, \tilde{E}_{j}\right)+\xi_{0} y_{0}^{2} \tilde{I}\left(\tilde{E}_{i}, \tilde{E}_{j}\right)=\xi_{0} \delta_{i j} .
$$

From this and the minimality of $\tilde{y}$, the mean curvature of $\tilde{x}: \tilde{M}^{n} \rightarrow \mathbb{S}^{n+1}$ is

$$
\tilde{H}=\frac{1}{n} \sum_{i=1}^{n} \tilde{h}_{i i}=\frac{y_{0}}{n} \sum_{i=1}^{p} \tilde{h}_{i i}^{*}+\xi_{0}=\xi_{0}
$$

Therefore, by definition, the Möbius factor $\tilde{\rho}$ of $\tilde{x}$ is determined by

$$
\tilde{\rho}^{2}=\frac{n}{n-1}\left(\sum_{i, j=1}^{n} \tilde{h}_{i j}^{2}-n \tilde{H}^{2}\right)=\frac{n}{n-1} y_{0}^{2} \sum_{i, j=1}^{p}\left(h_{i j}^{*}\right)^{2}=y_{0}^{2},
$$

where, in the last equality, we use $\sum_{i, j=1}^{p}\left(\tilde{h}_{i j}^{*}\right)^{2}=(n-1) / n$, which is implied by (3-42) and the Gauss equation of $\tilde{y}$. 
Hence, the Möbius position vector of $\tilde{x}$ is $\tilde{Y}=\tilde{\rho}(1, \tilde{x})=\left(y_{0}, y_{1}, y_{2}\right) \in \mathbb{L}^{n+3}$ and the Möbius metric of $\tilde{x}$ is

$$
\tilde{g}=\langle d \tilde{Y}, d \tilde{Y}\rangle=\left.\left(-d y_{0}^{2}+d y_{1}^{2}+d y_{2}^{2}\right)\right|_{\tilde{M}^{n}}=y_{0}^{2} \tilde{I} .
$$

Therefore, $\left\{\tilde{E}_{i}\right\}_{1 \leq i \leq n}$ is in fact a local orthonormal basis of the Möbius metric $\tilde{g}$. Furthermore, the Möbius second fundamental form of $\tilde{x}$ is

$$
\tilde{\mathbf{B}}=\tilde{\rho}^{-1} \sum_{i, j=1}^{n}\left(\tilde{h}_{i j}-\tilde{H} \delta_{i j}\right) \tilde{\omega}_{i} \tilde{\omega}_{j}=\sum_{i, j=1}^{p} \tilde{h}_{i j}^{*} \tilde{\omega}_{i} \tilde{\omega}_{j},
$$

where $\left\{\tilde{\omega}_{i}\right\}_{1 \leq i \leq n}$ is the dual basis of $\left\{\tilde{E}_{i}\right\}_{1 \leq i \leq n}$ on $\tilde{M}^{n}$. This is equivalent to

$$
\tilde{B}_{i j}= \begin{cases}\tilde{h}_{i j}^{*} & 1 \leq i, j \leq p \\ 0 & i>p \text { or } j>p .\end{cases}
$$

Since (4-53) shows that $\left(\tilde{M}^{n}, \tilde{g}\right)$ is the Riemannian direct product

$$
\left(\tilde{M}^{n}, \tilde{g}\right)=\left(N^{p}, d \tilde{y}^{2}\right) \times \mathbb{S}^{n-p}(r),
$$

we can use the Gauss equation to write down the Ricci tensor of $\tilde{g}$ with respect to $\left\{\tilde{E}_{i}\right\}_{1 \leq i \leq n}$ as

$$
\tilde{R}_{i j}= \begin{cases}-\frac{p-1}{r^{2}} \delta_{i j}-\sum_{k=1}^{p} \tilde{h}_{i k}^{*} \tilde{h}_{k j}^{*} & \text { if } 1 \leq i, j \leq p, \\ \frac{n-p-1}{r^{2}} \delta_{i j} & \text { if } p+1 \leq i, j \leq n, \\ 0, & \text { for all other cases, }\end{cases}
$$

which implies that the normalized scalar curvature $\tilde{R}$ of $\tilde{g}$ satisfies

$$
\begin{aligned}
n(n-1) \tilde{R} & =\frac{(n-p)(n-p-1)-p(p-1)}{r^{2}}-\sum_{i, j=1}^{p}\left(\tilde{h}_{i j}^{*}\right)^{2} \\
& =\frac{(n-p)(n-p-1)-p(p-1)}{r^{2}}-\frac{n-1}{n} .
\end{aligned}
$$

Thus

$$
\frac{1}{2 n}\left(1+n^{2} \tilde{R}\right)=\frac{(n-p)(n-p-1)-p(p-1)}{2(n-1) r^{2}} .
$$

From (2-12), (2-13) and (4-54)-(4-57), it follows that the Blaschke tensor of $\tilde{x}$ is given by $\tilde{\mathbf{A}}=\sum_{i, j=1}^{n} \tilde{A}_{i j} \tilde{\omega}_{i} \tilde{\omega}_{j}$, where the $\tilde{A}_{i j}$ form a diagonal matrix with entries $\tilde{A}_{i i}=-1 /\left(2 r^{2}\right)$ if $1 \leq i \leq p, \tilde{A}_{i i}=1 /\left(2 r^{2}\right)$ if $p+1 \leq i \leq n$, and 0 elsewhere. 
To show that $\tilde{x}: \tilde{M}^{n} \rightarrow \mathbb{S}^{n+1}$ has vanishing Möbius form, we can calculate directly according to its Equation (2-4). Nevertheless, compared with (4-51), it is not easy this time. Here is a simple argument proving $\tilde{\Phi}=0$ : On the Riemannian direct product $\left(\tilde{M}^{n}, \tilde{g}\right)=\left(N^{p}, d \tilde{y}^{2}\right) \times \mathbb{S}^{n-p}(r)$, the Blaschke tensor has two distinct constant eigenvalues as seen from the expression of $\tilde{\mathbf{A}}$, and their eigendistributions are both integrable with $\left(N^{p}, d \tilde{y}^{2}\right)$ and $\mathbb{S}^{n-p}(r)$ as their respective integrable manifolds. Then we easily see that the Blaschke tensor is also parallel. Now, according to [Li and Zhang 2006], we have $\tilde{\Phi}=0$.

Then, similarly to Proposition 4.2, if we compare the expression of $\tilde{\mathbf{A}}$ with (3-40) and (4-54) with (3-39), we can summarize the above calculation:

Proposition 4.5. If a hypersurface $\tilde{x}$ in $\mathfrak{C}_{2}$ is Möbius isoparametric, then must necessarily satisfy three conditions:

(i) $p=n-3$;

(ii) $r=\sqrt{6 n /(n-1)}$;

(iii) $\tilde{y}=\left(y_{0}, y_{1}\right): N^{n-3} \rightarrow \mathbb{M}^{n-2}(-(n-1) /(6 n))$ is a minimal isoparametric hypersurface with principal curvatures $\pm \sqrt{(n-1) /(2 n)}$ and 0 with multiplicity $n-5$.

On the other hand, according to E. Cartan [1938], an isoparametric hypersurface $M^{n}$ in hyperbolic space $\mathbb{T}^{n+1}$ can have at most two distinct principal curvatures, and $M^{n}$ must be either totally umbilic or else an open subset of a standard product $\mathbb{S}^{k} \times \mathbb{H}^{n-k}$ in $\mathbb{H}^{n+1}$; moreover, the later must be nonminimal. From this fact and Proposition 4.5, we have proved:

Proposition 4.6. There are no Möbius isoparametric hypersurfaces in $\mathfrak{C}_{2}$. More precisely, any hypersurface in $\mathfrak{C}_{2}$, if it exists, is of vanishing Möbius form, has two distinct, constant Blaschke eigenvalues, and nevertheless has nonconstant Möbius principal curvatures.

\section{Proof of the Main Theorem}

Let $x: M^{n} \rightarrow \mathbb{S}^{n+1}$ be a Möbius isoparametric hypersurface with three distinct Möbius principal curvatures, one of which is simple. Then we have two cases: if $x$ has parallel Möbius second fundamental form, then we apply Theorem 2.3 to obtain that it is locally Möbius equivalent to a hypersurface in (i) of the Main Theorem; if $x$ has nonparallel Möbius second fundamental form, then we can apply Theorem 3.7, Proposition 4.2, Remark 4.3 and Proposition 4.6 to conclude that it is locally Möbius equivalent to the hypersurface in (ii) of the Main Theorem.

Final Remark. For the general theory of Möbius submanifolds in $\mathbb{S}^{n+p}$ (see [Wang 1998]), the Möbius form $\Phi$ is an important invariant. In many interesting situations, 
we find that $\Phi=0$ is a very natural condition. For details, we refer to [Guo et al. 2001; Li et al. 2001; Liu et al. 2001; Li et al. 2002; 2003b; Li and Wang 2003a; Hu and Li 2003; 2004; 2005a; 2005b; Hu et al. 2007; Li and Zhang 2006; 2007], a series of nice results established in recent years under the condition $\Phi=0$.

\section{Acknowledgements}

The first author thanks his colleagues H. Li and C. P. Wang for helpful discussions and pleasant cooperation about topics on Möbius geometry of submanifolds and X. X. Li for his many valuable comments on the first version of this paper. Both authors are greatly indebted to [Li and Zhang 2007], which makes the present paper more easily accessible.

\section{References}

[Akivis and Goldberg 1996] M. A. Akivis and V. V. Goldberg, Conformal differential geometry and its generalizations, Pure and Applied Mathematics (New York), John Wiley \& Sons, New York, 1996. A Wiley-Interscience Publication. MR 98a:53023 Zbl 0863.53002

[Akivis and Goldberg 1997] M. A. Akivis and V. V. Goldberg, "A conformal differential invariant and the conformal rigidity of hypersurfaces", Proc. Amer. Math. Soc. 125:8 (1997), 2415-2424. MR 97j:53017 Zbl 0887.53030

[Cartan 1938] É. Cartan, "Familles de surfaces isoparamétriques dans les espaces à courbure constante", Ann. Mat. Pura Appl. 17:1 (1938), 177-191. MR 1553310 Zbl 0020.06505

[Cartan 1939] E. Cartan, "Sur des familles remarquables d'hypersurfaces isoparamétriques dans les espaces sphériques", Math. Z. 45 (1939), 335-367. MR 1,28f Zbl 0021.15603

[Cecil and Jensen 1998] T. E. Cecil and G. R. Jensen, "Dupin hypersurfaces with three principal curvatures", Invent. Math. 132:1 (1998), 121-178. MR 2000k:53051 Zbl 0908.53007

[Cecil and Jensen 2000] T. E. Cecil and G. R. Jensen, "Dupin hypersurfaces with four principal curvatures", Geom. Dedicata 79:1 (2000), 1-49. MR 2001g:53103 Zbl 0965.53039

[Guo et al. 2001] Z. Guo, H. Li, and C. Wang, "The second variational formula for Willmore submanifolds in $S^{n}$ ", Results Math. 40:1-4 (2001), 205-225. Dedicated to Shiing-Shen Chern on his 90th birthday. MR 2003b:53065 Zbl 01698602

[Hu and Li 2003] Z. Hu and H. Li, "Submanifolds with constant Möbius scalar curvature in $S^{n}$ ", Manuscripta Math. 111:3 (2003), 287-302. MR 2004c:53080 Zbl 1041.53007

[Hu and $\mathrm{Li} \mathrm{2004]} \mathrm{Z.} \mathrm{Hu} \mathrm{and} \mathrm{H}$. Li, "Classification of hypersurfaces with parallel Möbius second fundamental form in $S^{n+1}$ ", Sci. China Ser. A 47:3 (2004), 417-430. MR 2005c:53066 Zbl 1082.53016

[Hu and $\mathrm{Li}$ 2005a] Z. Hu and H. Li, "Classification of Möbius isoparametric hypersurfaces in $\mathbb{S}^{4}$ ", Nagoya Math. J. 179 (2005), 147-162. MR 2006e:53098 Zbl 05010498

[Hu and $\mathrm{Li} 2005 \mathrm{~b}] \mathrm{Z}$. Hu and $\mathrm{H}$. Li, "A rigidity theorem for hypersurfaces with positive Möbius Ricci curvature in $S^{n+1}$ ", Tsukuba J. Math. 29:1 (2005), 29-47. MR 2006g:53077 Zbl 05015594

[Hu et al. 2007] Z. Hu, H. Li, and C. Wang, "Classification of Möbius isoparametric hypersurfaces in $\mathbb{S}^{5}$.", Monatsh. Math. 151:3 (2007), 201-222. Zbl pre05180833

[Li and Wang 2003a] H. Li and C. Wang, "Möbius geometry of hypersurfaces with constant mean curvature and scalar curvature", Manuscripta Math. 112:1 (2003), 1-13. MR 2004e:53092 
[Li and Wang 2003b] H. Li and C. Wang, "Surfaces with vanishing Moebius form in $S^{n}$ ", Acta Math. Sin. (Engl. Ser.) 19:4 (2003), 671-678. MR 2004j:53076 Zbl 1078.53012

[Li and Zhang 2006] X. Li and F. Zhang, "A classification of immersed hypersurfaces in spheres with parallel Blaschke tensors”, Tohoku Math. J. (2) 58:4 (2006), 581-597. MR 2297201

[Li and Zhang 2007] X. Li and F. Zhang, "Immersed hypersurfaces in the unit sphere $S^{m+1}$ with constant Blaschke eigenvalues”, Acta Math. Sin., Engl. Ser. 23:3 (2007), 533-548. Zbl pre05162331

[Li et al. 2001] H. Li, C. Wang, and F. Wu, "A Moebius characterization of Veronese surfaces in $S^{n}$ ”, Math. Ann. 319:4 (2001), 707-714. MR 2002b:53098 Zbl 1031.53086

[Li et al. 2002] H. Li, H. Liu, C. Wang, and G. Zhao, "Möbius isoparametric hypersurfaces in $\mathbf{S}^{n+1}$ with two distinct principal curvatures", Acta Math. Sin. (Engl. Ser.) 18:3 (2002), 437-446. MR 2003h:53079 Zbl 1030.53017

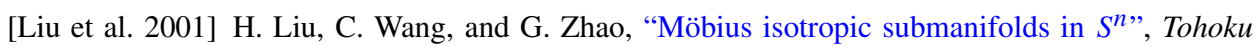
Math. J. (2) 53:4 (2001), 553-569. MR 2002f:53096 Zbl 1014.53010

[Niebergall 1991] R. Niebergall, "Dupin hypersurfaces in $\mathbf{R}^{5}$. I", Geom. Dedicata 40:1 (1991), 122. MR 92k:53106 Zbl 0733.53031

[Niebergall 1992] R. Niebergall, "Dupin hypersurfaces in $\mathbf{R}^{5}$. II", Geom. Dedicata 41:1 (1992), 5-38. MR 93b:53008 Zbl 0824.53008

[Pinkall 1985] U. Pinkall, "Dupinsche Hyperflächen in $E^{4 ”,}$ Manuscripta Math. 51:1-3 (1985), 89119. MR $86 \mathrm{~m}: 53010$

[Thorbergsson 1983] G. Thorbergsson, "Dupin hypersurfaces", Bull. London Math. Soc. 15 (1983), 493-498. MR 85b:53066 Zbl 0529.53044

[Wang 1998] C. Wang, "Moebius geometry of submanifolds in $S^{n}$ ", Manuscripta Math. 96:4 (1998), 517-534. MR 2000a:53019 Zbl 0912.53012

Received August 20, 2006. Revised December 22, 2006.

\section{ZEJUN HU}

DEPARTMENT OF MATHEMATICS

ZHENGZHOU UNIVERSITY

ZHENGZHOU 450052, HENAN

CHINA

huzj@zzu.edu.cn

DEYING LI

Department of Mathematics AND Physics

Pingdingshan Institute of TeChnOlogy

PINGDINGSHAN 467001, HENAN

CHINA

lideying@hncj.edu.cn 